Jani Onninen

\title{
Mappings of finite distortion: minors of the differential matrix
}

Received: 27 June 2003 / Accepted: 18 November 2003

Published online: 16 July 2004 - (C) Springer-Verlag 2004

\begin{abstract}
We extend the basic results on mappings of subexponentially integrable distortion to the cases where the usual distortion function is replaced by various weaker versions based on the minors of differential matrix.
\end{abstract}

Mathematics Subject Classification (2000): 30C65

\section{Introduction}

We consider mappings $f \in W_{\text {loc }}^{1,1}\left(\Omega, \mathbb{R}^{n}\right)$, where $\Omega \subset \mathbb{R}^{n}$ is a domain. Such a mapping is said to have finite distortion if

1. $f \in W_{\text {loc }}^{1,1}\left(\Omega, \mathbb{R}^{n}\right)$.

2. The Jacobian determinant $J(x, f)$ of $f$ is locally integrable.

3. There is a measurable function $K_{O}=K_{O}(x) \geq 1$, finite almost everywhere, such that $f$ satisfies the distortion inequality

$$
|D f(x)|^{n} \leq K_{O}(x) J(x, f) \quad \text { a.e. }
$$

Above we used the operator norm of the differential matrix. There are several distortion functions that are each of considerable interest in geometric function theory [6]. The principal feature of those distortions is, roughly speaking, that they provide some control on the lower order minors of the differential matrix in terms of the determinant. Let $\bigwedge^{l} f(x)$ denote the $\left(\begin{array}{c}n \\ l\end{array}\right) \times\left(\begin{array}{c}n \\ l\end{array}\right)$ - matrix of all $l \times l$-minors of $D f(x), l=1,2, \ldots, n-1$. Inequality (1) yields

$$
\left|\bigwedge^{l} f(x)\right|^{n} \leq \mathcal{K}_{l}(x)[J(x, f)]^{l} \quad \text { a.e. }
$$

J. Onninen: Department of Mathematics, University of Michigan, 525 East University Ann Arbor, MI 48109, USA

(e-mail: jonninen@umich.edu)

The author was supported in part by the Academy of Finland, project 39788, also supported by the foundations Magnus Ehrnroothin Säätiö and Vilho, Yrjö ja Kalle Väisälän Rahasto. This research was done when the author was visiting at the University of Michigan. He wishes to thank the department for the hospitality. 
where $1 \leq \mathcal{K}_{l}(x) \leq\left[K_{O}(x)\right]^{l}$. Notice that $\mathcal{K}_{1}(x)=K_{O}(x)$. On the other hand, notice that the assumptions $f \in W^{1, l}\left(B, \mathbb{R}^{n}\right), J(\cdot, f) \in L^{1}(B)$ and (2) with some $l \geq 2$ and $\mathcal{K}_{l}(x) \geq 1$, finite a.e., do not guarantee that $f$ be a mapping of finite distortion; consider e.g. $f\left(x_{1}, \ldots, x_{n}\right)=\left(x_{1}, 0, \ldots, 0\right)$. The smallest $\mathcal{K}_{l} \geq 1$ for which (2) holds will be denoted by $\mathcal{K}_{l}(x, f)$ and called the $l$-th distortion function. Of particular interest is the inner distortion function $K_{I}(x, f)=\mathcal{K}_{n-1}(x, f)$. In this case we denote by $D^{\sharp} f(x)$ the $n \times n$-matrix of cofactors of $D f$. Thus (2) reads as

$$
\left|D^{\sharp} f(x)\right|^{\frac{n}{n-1}} \leq \sqrt[n-1]{K_{I}(x, f)} J(x, f) .
$$

In this paper we prove the following continuity estimate.

Theorem 1.1. Let $n \geq 2$ and $l \in\{1, \ldots, n-1\}$. Assume that $f \in W_{l o c}^{1, l}\left(B, \mathbb{R}^{n}\right)$ is a mapping of finite distortion such that

$$
I=\int_{B} \exp \left(\lambda \sqrt[l]{\mathcal{K}_{l}(x, f)}\right) d x<\infty
$$

for some $\lambda>l^{2}-1$ and a ball $B=B\left(x_{0}, R\right)$. Then $f$ is continuous and we have the modulus of continuity estimate

$$
|f(x)-f(y)|^{n} \leq \frac{C_{\lambda, K, R, n}(\epsilon) \int_{B} J(z, f) d z}{\log ^{\lambda-l^{2}+1-\epsilon}\left(\frac{n I}{\omega_{n-1}|x-y|^{n}}\right)}
$$

for all $x, y \in B\left(x_{0},(R / 8)^{e}\left[\frac{n I}{\omega_{n-1}}\right]^{\frac{1-e}{n}}\right)$ and every small $\epsilon>0$.

If $n \geq 3$, then the example $f(x)=(u(x), 0, \ldots, 0)$, where $u$ is a discontinuous function in the Sobolev space $W^{1, n-1}(B)$, shows that the assumption that $f$ be of finite distortion cannot be dropped from Theorem 1.1. We mean that we cannot replace the distortion inequality (1) by the the weaker distortion inequality (2), for any $l \in\{2, \ldots, n-1\}$. We do not know if the bound $\lambda>l^{2}-1$ is necessary. For $l=1$, our estimate is contained in [12] but the case $l \geq 2$ is new. If one tries to reduce the case $l \geq 2$ to $l=1$, one is faced with the following obstacle. By pointwise estimates one can only guarantee the integrability of $\exp \left(\lambda K_{O}^{1 / l^{2}}\right)$ which is, in general, too weak to imply continuity [10]. Our proof is based on an improvement of our argument in [12].

Theorem 1.1 leads to compactness results via the Ascoli's Theorem and our compactness result in [5].

Theorem 1.2. Fix $l \in\{1, \ldots, n-1\}, \lambda>l^{2}-1$ and $A, B \geq 0$. Let $\mathcal{F}$ be the family of mappings $f: \Omega \rightarrow \mathbb{R}^{n}$ of finite distortion for which

$$
\int_{\Omega} J(x, f) d x \leq A
$$

and

$$
\int_{\Omega} \exp \left(\left(\lambda \sqrt[l]{\mathcal{K}_{l}(x, f)}\right)\right) d x \leq B
$$


Fix $x_{0} \in \Omega$ and define $\tilde{\mathcal{F}}=\left\{g: g(x)=f(x)-f\left(x_{0}\right)\right.$ and $\left.f \in \mathcal{F}\right\}$. Then each sequence of mappings in $\tilde{\mathcal{F}}$ contains a locally uniformly converging subsequence, and the limit of any such a sequence belongs to $\tilde{\mathcal{F}}$.

Furthermore, by combining Theorem 1.1 with results by Kauhanen, Koskela and Malý (cf. [8] and [9]), we can conclude topological properties, such as openness and discretness and also an analytic property, the Lusin condition $(N)$. Openness means that $f$ maps open sets to open sets and discretness that the set of preimages of any point in $\mathbb{R}^{n}$ is finite in each compact subset of $\Omega$. The Lusin condition $(N)$ means that $f$ maps sets of measure zero to sets of measure zero.

Theorem 1.3. Fix $l \in\{1, \ldots, n-1\}, \lambda>l^{2}-1$. Assume that $f \in W_{l o c}^{1, l}\left(\Omega, \mathbb{R}^{n}\right)$ is a mapping of finite distortion such that $\exp \left(\left(\lambda \sqrt[l]{\mathcal{K}_{l}(x, f)}\right)\right) \in L_{\text {loc }}^{1}(\Omega)$. Then $f$ satisfies the Lusin condition $(N)$ and if $f$ is non-constant, then it is also open and discrete.

\section{Distortion functions}

Distortion functions are designed to control almost everywhere the minors of the differential matrix of the mapping $f: \Omega \rightarrow \mathbb{R}^{n}$ by means of the Jacobian determinant. We begin with the distortion functions of linear mappings, also regarded as matrices. The space of all $n \times n$ matrices will be denoted by $\mathbb{R}^{n \times n}$, and those with positive determinant by $\mathbb{R}_{+}^{n \times n}$. It will be convenient to include the zero matrix and denote such extended class of matrices by $\mathbb{R}_{+}^{n \times n} \cup\{0\}$. that The commonly used distortion functions on matrices $A \in \mathbb{R}_{+}^{n \times n}$ are:

The outer distortion

$K_{O}(A)=\frac{|A|^{n}}{\operatorname{det} A}$.

The inner distortion

$K_{I}(A)=K_{O}\left(A^{-1}\right)=\frac{\left|A^{\sharp}\right|^{n}}{(\operatorname{det} A)^{n-1}}$.

The linear distortion

$H(A)=\sqrt[n]{K_{O}(A) K_{I}(A)}=|A|\left|A^{-1}\right|$.

Note that the operator norm $|A|=\max \{|A h|:|h|=1\}$ is being used here, and $A^{\sharp}$ is the adjoint matrix, made of cofactors of $A$. In what follows all distortion functions of the zero matrix are assumed to be equal to 1 . There are in fact many more distortion functions which are readily defined in terms of the lower order subdeterminants of the matrix $A$. For each integer $1 \leq l \leq n$ we denote by $\bigwedge^{l} A$ the $\left(\begin{array}{l}n \\ l\end{array}\right) \times\left(\begin{array}{l}n \\ l\end{array}\right)$ matrix of all $l \times l$-minors of $A$. This, of course, includes $A$ as $\bigwedge^{1} A$, $A^{\sharp}$ as $\bigwedge^{n-1} A$, and $\operatorname{det} A$ as $\bigwedge^{n} A$. The following distortion functions will be of interest to us:

$$
\mathcal{K}_{l}(A)=\frac{\left|\bigwedge^{l} A\right|^{n}}{(\operatorname{det} A)^{l}}=\mathcal{K}_{n-l}\left(A^{-1}\right) \text { for } l=1, \ldots, n-1 .
$$

Having examined these distortion functions for matrices we set for orientation preserving mappings (i.e. $J(x, f) \geq 0$ a.e.) $f \in W_{l o c}^{1,1}\left(\Omega, \mathbb{R}^{n}\right)$ :

$$
\bigwedge^{l} f(x)=\bigwedge^{l}[D f(x)]
$$


We define the point wise distortion functions by setting

$$
\mathcal{K}_{l}(x, f)=\mathcal{K}_{l}[D f(x)]= \begin{cases}\frac{\left|\bigwedge^{l} f(x)\right|^{n}}{J(x, f)^{l}}, & J(x, f)>0 \\ 1, & \left|\bigwedge^{l} f(x)\right|=0 \\ \infty, & J(x, f)=0 \text { and }\left|\bigwedge^{l} f(x)\right| \neq 0 .\end{cases}
$$

These functions are coupled by the inequalities

$$
\sqrt[n-1]{K_{I}(x, f)}=\sqrt[n-1]{\mathcal{K}_{n-1}(x, f)} \leq \ldots \leq \sqrt[l]{\mathcal{K}_{l}(x, f)} \leq \mathcal{K}_{1}(x, f)=K_{O}(x, f) .
$$

Let us also note for later use the reverse estimate

$$
K_{O}(x, f) \leq \mathcal{K}_{l}^{l}(x, f)=\left(\sqrt[l]{\mathcal{K}_{l}(x, f)}\right)^{l^{2}}
$$

that holds when $J(x, f)>0$.

\section{Monotonicity}

A very powerful method when dealing with continuity properties of functions is furnished by notion of monotonicity, which goes back to H. Lebesgue [13] in 1907. Monotonicity for a continuous function $u$ in a domain $\Omega$ simply means that

$$
\operatorname{osc}(u, B) \leq \operatorname{osc}(u, \partial B)
$$

for every ball $B \subset \Omega$. It turns out that monotonicity can be defined without the continuity assumption. The following definition is due to J. Manfredi [14].

Definition 3.1. A real valued function $u \in W^{1,1}(\Omega)$ is said to be weakly monotone if for every ball $B \subset \Omega$ and all constants $m \leq M$ such that

$$
(u-M)^{+}-(m-u)^{+} \in W_{0}^{1,1}(B)
$$

we have

$$
m \leq u(x) \leq M
$$

for almost every $x \in B$.

The space $W_{0}^{1,1}(B)$ is the completion of $C_{0}^{\infty}(B)$ in $W^{1,1}(B)$, as usually. The following proposition states the weak monotonicity of a coordinate function of $f$, under assumptions which are adapted to our situation. This is based on the fact that $J(\cdot, f)$ coincides in this setting with the distributional Jacobian, i.e.

$$
\int_{\Omega} \varphi(x) J(x, f) d x=-\int_{\Omega} f_{i} J\left(x, f_{1}, \ldots, f_{i-1}, \varphi, f_{i+1}, \ldots, f_{n}\right)
$$

for all test functions $\varphi \in C_{0}^{\infty}(\Omega)$ and each index $i=1,2, \ldots, n$. 
Proposition 3.2. Let $f \in W_{\text {loc }}^{1, l}\left(\Omega, \mathbb{R}^{n}\right)$ be a mapping of finite distortion such that $\exp \left(\lambda \sqrt[l]{\mathcal{K}_{l}(\cdot, f)}\right) \in L_{l o c}^{1}(\Omega)$ for some $\lambda>0$ and some $l \in\{1, \ldots, n-1\}$. Then $f \in W_{\text {loc }}^{1, p}\left(\Omega, \mathbb{R}^{n}\right)$, for all $p \in[1, n),\left|D^{\sharp} f\right|^{\frac{n}{n-1}} \log ^{-1}\left(e+\left|D^{\sharp} f\right|\right) \in L_{\text {loc }}^{1}(\Omega)$, the equation (16) holds, and the coordinates functions of $f$ are weakly monotone.

Proof. Fix $p \in[1, n)$ and $\Omega^{\prime}$. Then by Hölder's inequality we have

$$
\left(\int_{\Omega^{\prime}}|D f|^{p}\right)^{\frac{n}{p}} \leq\left(\int_{\Omega^{\prime}} K_{O}^{\frac{p}{n-p}}(x) d x\right)^{\frac{n-p}{p}} \int_{\Omega^{\prime}} J(x, f) d x .
$$

Using the power series presentation for the function $\exp \left(\lambda K_{O}^{1 / l^{2}}\right)$, inequality (12) and inequality (17), we see that $f \in W^{1, p}\left(\Omega^{\prime}, \mathbb{R}^{n}\right)$. Recall here that $D f$ vanishes a.e. in the zero set of the Jacobian.

The point of special note is that our assumptions imply

$$
\left|D^{\sharp} f(x)\right|^{n} \leq K_{I}(x, f) J(x, f)^{n-1}
$$

with

$$
\int_{\Omega^{\prime}} \exp \left(\lambda \sqrt[n-1]{K_{I}(x, f)}\right) d x<\infty
$$

(see our estimate (11)). Combining Hadamard's inequality $J(x, f) \leq\left|D^{\sharp} f(x)\right|^{\frac{n}{n-1}}$ with (18) and with the elementary inequality $a b \leq a \log (a+1)+e^{b}-1$ for $a, b \geq 0$, we have

$$
\begin{aligned}
& \frac{n-1}{n} \int_{\Omega^{\prime}} \frac{\left|D^{\sharp} f(x)\right|^{\frac{n}{n-1}} d x}{\log \left(e+\left|D^{\sharp} f(x)\right|\right)} \leq \int_{\Omega^{\prime}} \frac{\left|D^{\sharp} f(x)\right|^{\frac{n}{n-1}} d x}{\log \left(e+\left|D^{\sharp} f(x)\right|^{\frac{n}{n-1}}\right)} \\
& \leq \frac{1}{\lambda} \int_{\Omega^{\prime}} J(x, f) d x+\int_{\Omega^{\prime}} \exp \left(\lambda \sqrt[n-1]{K_{I}(x, f)}\right) d x \\
& <\infty \text {. }
\end{aligned}
$$

This and the fact $f \in W_{l o c}^{1, n-1}(\Omega)$ imply, via recent results in [1, Theorem 1.3], that we can integrate by parts against the Jacobian, i.e

$$
\int_{\Omega} \varphi(x) J(x, f) d x=-\int_{\Omega} f_{i} J\left(x, f_{1}, \ldots, f_{i-1}, \varphi, f_{i+1}, \ldots, f_{n}\right)
$$

for all test functions $\varphi \in C_{0}^{\infty}(\Omega)$ and each index $i=1,2, \ldots, n$.

Next we follow the idea from $[4$, Sect. 4$]$ to prove that the coordinates functions of $f$ are weakly monotone. Let $\tilde{B}$ be a ball in $B$ and suppose that for some coordinate function, say the first one, we have

$$
v:=\left(f_{1}-M\right)^{+}-\left(m-f_{1}\right)^{+} \in W_{0}^{1,1}(B) .
$$

Then

$$
\nabla v(x)= \begin{cases}0 & \text { if } m \leq f^{1}(x) \leq M \\ \nabla f_{1}(x), & \text { otherwise (say, on the set } E \subset \tilde{B}) .\end{cases}
$$


Hence, in view of the distortion inequality (1), we can write

$$
\begin{aligned}
\int_{\tilde{B}} \frac{|\nabla v(x)|^{n}}{K(x)} d x & \leq \int_{E} \frac{|D f(x)|^{n}}{K(x)} d x \\
& \leq \int_{E} J(x, f) d x \leq \int_{\tilde{B}} J\left(x, v, f_{2}, \ldots, f_{n}\right) d x=0
\end{aligned}
$$

by equation (21). Thus $v \equiv 0$ on $\tilde{B}$, which simply means that $m \leq f_{1}(x) \leq M$ for almost every $x \in \tilde{B}$, as desired.

\section{Proof of Theorem 1.1}

We will split the proof of Theorem 1.1 to two parts, Lemma 4.1 and Lemma 4.3.

Lemma 4.1. Let $f: \Omega \rightarrow \mathbb{R}^{n}$ be a mapping in the Sobolev class $W_{\text {loc }}^{1, n-1}\left(\Omega, \mathbb{R}^{n}\right)$ such that $J(\cdot, f) \in L_{\text {loc }}^{1}(\Omega)$ and

$$
\left|D^{\sharp} f(x)\right|^{\frac{n}{n-1}} \leq \sqrt[n-1]{K_{I}(x)} J(x, f) \text { a.e. }
$$

with

$$
I=\int_{B} \exp \left(\lambda \sqrt[n-1]{K_{I}(x)}\right) d x<\infty
$$

for some $\lambda>0$ and a ball $B=B\left(x_{0}, R\right) \subset \subset \Omega$. Then for every small $\epsilon>0$, we have

$$
\int_{B\left(x_{0}, r\right)} J(x, f) d x \leq \frac{C_{\lambda, K, R, n}(\epsilon) \int_{B} J(z, f) d z}{\log ^{\lambda-\epsilon}\left(\frac{n I}{\omega_{n-1} r^{n}}\right)}
$$

whenever $r \in(0, R / 2)$.

The proof of Lemma 4.1 will be based on the following integral type isoperimetric inequality, established in [16] (also see [4]).

Proposition 4.2. Suppose that the Jacobian of $f \in W_{l o c}^{\frac{n^{2}}{n+1}}\left(\Omega, \mathbb{R}^{n}\right)$ is non-negative a.e and the mapping $f$ obeys the rule (16) of integration by parts. Then, for every $x_{0} \in \Omega$, we have

$$
\int_{B\left(x_{0}, r\right)} J(x, f) d x \leq\left(n \sqrt[n-1]{\omega_{n-1}}\right)^{-1}\left(\int_{\partial B\left(x_{0}, r\right)}\left|D^{\sharp} f\right|\right)^{\frac{n}{n-1}}
$$

for almost every radius $r \in\left(0, \operatorname{dist}\left(x_{0}, \partial \Omega\right)\right)$.

The basic idea of the proof of Lemma 4.1 goes back to Morrey [15], we follow the ideas from [12]. 
Proof. Using Proposition 3.2 we see that the assumptions of Proposition 4.2 are fulfilled, and so

$$
\int_{B\left(x_{0}, s\right)} J(x, f) d x \leq\left(n \sqrt[n-1]{\omega_{n-1}}\right)^{-1}\left(\int_{\partial B\left(x_{0}, s\right)}\left|D^{\sharp} f\right|\right)^{\frac{n}{n-1}}
$$

for almost every $0<s<R$. Write $B_{s}=B\left(x_{0}, s\right)$. Fix $\epsilon \in(0,1)$ and $i \in$ $\{1,2,3, \ldots\}$. We denote the interval $\left(R 2^{-\epsilon i}, R 2^{-\epsilon(i-1)}\right)$ by $\triangle_{i, \epsilon}$ and the annulus $B_{R 2^{-\epsilon(i-1)}} \backslash B_{R 2^{-\epsilon i}}$ by $A_{i, \epsilon}$. Using Fubini's theorem we observe that for every $\delta>0$ the set

$$
E_{i}=\left\{t \in \triangle_{i, \epsilon}: \int_{\partial B_{t}}\left|D^{\sharp} f\right| \leq \frac{(1+\delta)}{\left|\triangle_{i, \epsilon}\right|} \int_{A_{i, \epsilon}}\left|D^{\sharp} f(x)\right| d x\right\}
$$

has a positive measure.

Choosing $r \in \triangle_{i, \epsilon}$ so that $r$ lies in the set $E_{i}$ and so that inequality (26) holds, we obtain the estimate

$$
\begin{aligned}
\int_{B_{R 2}-\epsilon i} J(x, f) d x & \leq \int_{B_{r}} J(x, f) d x \leq\left(n \sqrt[n-1]{\omega_{n-1}}\right)^{-1}\left(\int_{\partial B_{r}}\left|D^{\sharp} f\right|\right)^{\frac{n}{n-1}} \\
& \leq\left(n \sqrt[n-1]{\omega_{n-1}}\right)^{-1}\left(\frac{1+\delta}{\left|\triangle_{i, \epsilon}\right|}\right)^{\frac{n}{n-1}}\left(\int_{A_{i, \epsilon}}\left|D^{\sharp} f(x)\right| d x\right)^{\frac{n}{n-1}}
\end{aligned}
$$

We set $t_{n}(\lambda)=\left(\frac{n-2}{\lambda}\right)^{n-1}$ and

$$
\tilde{K}_{I}(x)= \begin{cases}K_{I}(x), & K_{I}(x)>t_{n}(\lambda) \\ t_{n}(\lambda), & K_{I}(x) \leq t_{n}(\lambda) .\end{cases}
$$

Combining the distortion inequality $\left|D^{\sharp} f(x)\right| \leq \tilde{K}_{I}^{\frac{1}{n}}(x) J(x, f)^{\frac{n-1}{n}}$ with Hölder's inequality, we find that

$$
\begin{aligned}
\int_{B_{R 2}-\epsilon i} J(x, f) d x \leq & \left(n \sqrt[n-1]{\omega_{n-1}}\right)^{-1}\left(\frac{1+\delta}{\left|\triangle_{i, \epsilon}\right|}\right)^{\frac{n}{n-1}} \\
& \left(\int_{A_{i, \epsilon}}\left|\tilde{K}_{I}\right|\right)^{\frac{1}{n-1}} \int_{A_{i, \epsilon}} J(x, f) d x .
\end{aligned}
$$

Jensen's inequality applied to the convex function $\left(t_{n}(\lambda), \infty\right) \rightarrow(0, \infty): \tau \rightarrow$ $\exp \left(\lambda \tau^{1 /(n-1)}\right)$ yields

$$
\begin{aligned}
\int_{B_{R 2}-\epsilon i} J(x, f) d x \leq & \left(n \sqrt[n-1]{\omega_{n-1}}\right)^{-1}\left(\frac{1+\delta}{\left|\triangle_{i, \epsilon}\right|}\right)^{\frac{n}{n-1}} \frac{\left|A_{i, \epsilon}\right|^{\frac{1}{n-1}}}{\lambda} \\
& \log \left(\int_{A_{i, \epsilon}} \exp \left(\lambda \sqrt[n-1]{K_{I}}\right)\right) \int_{A_{i, \epsilon}} J(x, f) d x
\end{aligned}
$$


and computations show that

$$
\begin{aligned}
\int_{B_{R 2}-\epsilon i} J(x, f) d x \leq & \left(\frac{1+\delta}{n}\right)^{\frac{n}{n-1}}\left(\frac{2^{\epsilon n}-1}{2^{\epsilon}-1}\right)^{\frac{1}{n-1}} R 2^{-\epsilon i}\left|\triangle_{i, \epsilon}\right|^{-1} \\
& \log \left(\frac{\exp \left(\lambda \sqrt[n-1]{t_{n}(\lambda)}\right) n I 2^{\epsilon n}}{\omega_{n-1} R^{n} 2^{-\epsilon n(i-1)}\left(2^{\epsilon n}-1\right)}\right) \int_{A_{i, \epsilon}} J(x, f) d x
\end{aligned}
$$

Write $C_{\lambda}(n)=\exp \left(\lambda \sqrt[n-1]{t_{n}(\lambda)}\right) 2^{n}$. Letting $\delta \rightarrow 0$, using the elementary inequality $\epsilon a \log 2 \leq 2^{a \epsilon}-1 \leq \epsilon a 2^{a \epsilon} \log 2$ for all $\epsilon, a \geq 0$, and the fact that $\epsilon \leq 1$, we conclude that

$$
\begin{aligned}
\int_{B_{R 2}-\epsilon i} J(x, f) d x & \leq \frac{2^{\frac{n \epsilon}{n-1}}}{\lambda n} R 2^{-\epsilon i} \log \left(\frac{C_{\lambda}(n) n I}{\omega_{n-1} R^{n} 2^{-\epsilon n(i-1)} \epsilon}\right) \\
& \times \int_{A_{i, \epsilon}} J(x, f) d x\left|\triangle_{i, \epsilon}\right|^{-1} .
\end{aligned}
$$

Next we introduce two auxiliary functions. We set

$$
u_{i, \epsilon}(x)=\int_{B_{R 2}-\epsilon i} J(z, f) d z+\frac{x-R 2^{-\epsilon i}}{\left|\triangle_{i, \epsilon}\right|} \int_{A_{i, \epsilon}} J(z, f) d z
$$

and

$$
u_{\epsilon}(x)=\sum_{i=1}^{\infty} u_{i, \epsilon}(x) \chi_{\left[R 2^{-\epsilon i}, R 2^{-\epsilon(i-1)}\right)}(x)
$$

for every $x \in \mathbb{R}$. Using inequality (30), we see that

$$
u_{i, \epsilon}(x) \leq\left[\frac{2^{\frac{n \epsilon}{n-1}}}{\lambda n} R 2^{-\epsilon i} \log \left(\frac{C_{\lambda}(n) n I}{\omega_{n-1} R^{n} 2^{-\epsilon n(i-1)} \epsilon}\right)+x-R 2^{-\epsilon i}\right] u_{i, \epsilon}^{\prime}(x)
$$

for all $x \in \triangle_{i, \epsilon}$. Combining the inequality $R 2^{-\epsilon(i-1)}-R 2^{-\epsilon i} \leq R 2^{-\epsilon i} 2 \epsilon$ with the fact that

$$
\begin{aligned}
\log ^{-1}\left(\frac{C_{\lambda(n)} n I}{\omega_{n-1} R^{n} 2^{-\epsilon n(i-1)} \epsilon}\right) & \leq \log ^{-1}\left(\frac{2^{\epsilon n} C_{\lambda}(n) n I}{\epsilon \omega_{n-1} R^{n}}\right) \\
& \leq \log ^{-1}\left(\frac{2^{\epsilon n} C_{\lambda}(n)}{\epsilon}\right) \leq C_{1}(n, \lambda)
\end{aligned}
$$

for all $\epsilon \in(0,1)$, we conclude with

$$
u_{i, \epsilon}(x) \leq\left(\frac{2^{\frac{n \epsilon}{n-1}}}{\lambda n}+2 C_{1}(n, \lambda) \epsilon\right) x \log \left(\frac{C_{\lambda}(n) n I}{\omega_{n-1} x^{n} \epsilon}\right) u_{i, \epsilon}^{\prime}(x)
$$

for all $x \in \triangle_{i, \epsilon}$.

For shorter the notation, we write

$$
\beta(\epsilon)=\frac{2^{\frac{n \epsilon}{n-1}}}{\lambda n}+2 C_{1}(n, \lambda) \epsilon
$$


and

$$
\alpha(\epsilon)=\frac{C_{\lambda}(n) n I}{\omega_{n-1} \epsilon} .
$$

We define

$$
v_{\epsilon}(x)=\left[\log \left(\frac{\alpha(\epsilon)}{x^{n}}\right)\right]^{\frac{1}{n \beta(\epsilon)}}
$$

for all $x \in(0, R)$. Using inequality (32), we see that

$$
\frac{d}{d x}\left(v_{\epsilon} u_{\epsilon}\right)(x)=v_{\epsilon}(x)\left[-\left(\beta(\epsilon) x \log \left(\frac{\alpha(\epsilon)}{x^{n}}\right)\right)^{-1} u_{\epsilon}(x)+u_{\epsilon}^{\prime}(x)\right] \geq 0
$$

for all $x \in \cup_{i=1}^{\infty} \triangle_{i, \epsilon}$, and so the continuous function $v_{\epsilon} u_{\epsilon}$ is increasing on $(0, R)$. Let $r \leq R / 2$. Choosing $i_{r} \in \mathbb{N}$ so that $r \in\left[R 2^{-\epsilon i_{r}}, R 2^{-\epsilon\left(i_{r}-1\right)}\right)$, we find that

$$
v_{\epsilon, \delta}\left(R 2^{-\epsilon i_{r}}\right) \int_{B_{R 2}-\epsilon i_{r}} J(x, f) d x \leq 2 v_{\epsilon, \delta}(R-\delta) \int_{B_{R-\delta}} J(x, f) d x .
$$

Since the Jacobian of $f$ is non-negative almost everywhere and $\epsilon \leq 1$, we find that

$$
\int_{B_{r}} J(x, f) d x \leq 2 \frac{v_{\epsilon}(R)}{v_{\epsilon}(2 r)} \int_{B_{R}} J(x, f) d x
$$

for all $0<r<R / 2$. Using the fact that $n \beta(\epsilon) \rightarrow \frac{1}{\lambda}$, as $\epsilon \rightarrow 0$, we obtain the desired inequality (24).

Theorem 1.1 is proven in the case $l=1$ in [12, Theorem 1.1]. So we will assume that $n>2$ and $l \in\{2, \ldots, n-1\}$.

Lemma 4.3. Let $n>2$ and $l \in\{2, \ldots, n-1\}$. Assume that $f: \Omega \rightarrow \mathbb{R}^{n}$ is a mapping of finite distortion such that (4) holds, for some $\lambda>0$ and a ball $B_{r}=B\left(x_{0}, r\right) \subset \subset \Omega$. Then

$$
|\hat{f}(x)-\hat{f}(y)|^{n} \leq C_{\lambda, n, l} \log ^{l^{2}-1}\left(\frac{n I}{\omega_{n-1} r^{n}}\right) \int_{B_{r}} J(z, f) d z
$$

for all $x, y \in B\left(x_{0},(r / 2)^{e}\left[\frac{n I}{\omega_{n-1}}\right]^{\frac{1-e}{n}}\right)$.

Here

$$
I=\int_{B_{r}} \exp \left(\lambda \sqrt[l]{\mathcal{K}_{l}(x, f)}\right) d x
$$

we used the representative $\hat{f}$ for the mapping $f$, defined by setting $\hat{f}=\left(\hat{f}_{1}, \ldots, \hat{f}_{n}\right)$ and

$$
\hat{f}_{i}(x)=\lim _{r \rightarrow 0} \sup \int_{B(x, r)} f_{i}(z) d z
$$

for all $i \in\{1, \ldots, n\}$. 
Proof. Let $p=n-\frac{1}{2}$ and write $\varphi(r)=(r / 2)^{e}\left[\frac{n I}{\omega_{n-1}}\right]^{\frac{1-e}{n}}$. Combining Proposition 3.2 with Lemma 7.2 in [4] we have

$$
\frac{|\hat{f}(x)-\hat{f}(y)|^{n}}{C(p, n) t^{n}} \leq\left(\int_{\partial B\left(x_{0}, t\right)}|D f|^{p}\right)^{\frac{n}{p}}
$$

for almost every $t \in(\varphi(r), r)$ and all $x, y \in B\left(x_{0}, \varphi(r)\right)$. We would like to point out here that the estimate (39) goes back to the oscillation lemma by F. W. Gehring [2].

Write $B_{s}=B\left(x_{0}, s\right)$ for all $s \in(0, r)$ and define

$$
G_{i}=\left\{t \in\left(2^{i-1} \varphi(r), 2^{i} \varphi(r)\right): \int_{\partial B_{t}} \exp \left(\lambda K_{O}^{1 / l^{2}}\right) \leq \frac{3}{2^{i-1} \varphi(r)} \int_{A_{i}} \exp \left(\lambda K_{O}^{1 / l^{2}}\right)\right\}
$$

for all $i \in\{1,2, ..\} \cap\left[1, \log _{2} \frac{r}{\varphi(r)}\right]=: S$. Here and also in what follows we denote the set $B_{2^{i} \varphi(r)} \backslash B_{2^{i-1} \varphi(r)}$ by $A_{i}$. Because $I \geq \frac{\omega_{n-1}}{n} r^{n}$, we see that $S \neq \emptyset$. Using estimate (12) we remark that

$$
\int_{A_{i}} \exp \left(\lambda K_{O}^{1 / l^{2}}\right) \leq \int_{A_{i}} \exp \left(\lambda \sqrt[l]{\mathcal{K}_{l}}\right)<\infty
$$

By Fubini's theorem, we have

$$
\left|G_{i}\right| \geq \frac{2^{i-1} \varphi(r)}{2}
$$

Next we fix $t_{0}=t_{0}(p, n, l, \lambda)$ so that $t_{0}^{\frac{p l^{2}}{\lambda(n-p)}} \geq \frac{p l^{2}}{\lambda(n-p)}$; then the function $t \rightarrow$ $\exp \left(\lambda t^{\frac{n-p}{p l^{2}}}\right)$ is convex on $\left(t_{0}, \infty\right)$. We set

$$
\tilde{K}_{O}(x)= \begin{cases}K_{O}(x), & K_{O}(x)>t_{0} \\ t_{0}, & K_{O}(x) \leq t_{0}\end{cases}
$$

Combining the distortion inequality $|D f(x)|^{n} \leq \tilde{K}_{O}(x) J(x, f)$ and Hölder's inequality with inequality (39), we have

$$
\frac{|\hat{f}(x)-\hat{f}(y)|^{n}}{C(p, n) t^{n}} \leq\left(\int_{\partial B_{t}}\left|\tilde{K}_{O}(x)\right|^{\frac{p}{n-p}}\right)^{\frac{n-p}{p}} f_{\partial B_{t}} J(x, f) d x
$$

for a.e. $t \in(\varphi(r), r)$ and all $x, y \in B_{\varphi(r)}$. Jensen's inequality applied to the convex function $\left(t_{0}, \infty\right) \rightarrow(0, \infty): \tau \rightarrow \exp \left(\lambda \tau^{\frac{n-p}{p l^{2}}}\right)$ yields

$$
\begin{aligned}
\frac{|\hat{f}(x)-\hat{f}(y)|^{n}}{C(p, n) t} & \leq \lambda^{\frac{p l^{2}}{n-p}} \log ^{l^{2}}\left(\int_{\partial B_{t}} \exp \left(\lambda \tilde{K}_{O}^{1 / l^{2}}\right)\right) \int_{\partial B_{t}} J(x, f) d x \\
& \leq \lambda^{\frac{p l^{2}}{n-p}} \log ^{l^{2}}\left(\exp \left(\lambda t_{0}^{1 / l^{2}}\right) \int_{\partial B_{t}} \exp \left(\lambda K_{O}^{1 / l^{2}}\right)\right) \int_{\partial B_{t}} J(x, f) d x
\end{aligned}
$$


for a.e. $t \in(\varphi(r), r)$ and all $x, y \in B_{\varphi(r)}$. Fix $i \in S$. Integrating this estimate over the set $G_{i}$ with respect to $t$, we have

$$
|\hat{f}(x)-\hat{f}(y)|^{n} \int_{G_{i}} \log ^{-l^{2}}\left(\frac{\exp \left(\lambda t_{0}^{1 / l^{2}}\right) 6 n I}{\omega_{n-1} 2^{i} t^{n-1} \varphi(r)}\right) \frac{d t}{t} \leq C_{\lambda}(p, n) \int_{A_{i}} J(z, f) d z
$$

for every $x, y \in B_{\varphi(r)}$. Here we also used the estimate

$$
\int_{A_{i}} \exp \left(\lambda K_{O}^{\frac{1}{l^{2}}}\right) \leq \int_{B_{r}} \exp \left(\lambda \sqrt[l]{\mathcal{K}_{l}}\right)=I
$$

For every $t \in G_{i}$, we have $t \leq 2^{i} \varphi(r)$ and so

$$
|\hat{f}(x)-\hat{f}(y)|^{n} \int_{G_{i}} \log ^{-l^{2}}\left(\frac{\exp \left(\lambda t_{0}^{1 / l^{2}}\right) 6 n I}{\omega_{n-1} 2^{i} t^{n-1} \varphi(r)}\right) \frac{d t}{t} \leq C_{\lambda}(p, n) \int_{A_{i}} J(z, f) d z
$$

for every $x, y \in B_{\varphi(r)}$. Replacing the constant $6 \exp \left(\lambda t_{0}^{1 / l^{2}}\right)$ in (44) by the constant $C_{\lambda}\left(t_{0}\right)=\max \left\{e^{n l^{2}}, \exp \left(\lambda t_{0}^{1 / l^{2}}\right) 6\right\}$ we see that the function $t \log ^{l^{2}}\left(\frac{C_{\lambda}\left(t_{0}\right) n I}{t^{n} n}\right)$ is increasing on $(0, R)$ (Here we used the fact $I \geq \frac{\omega_{n-1}}{n} r^{n}$ ). Combining this with estimate (44) and the fact $\left|G_{i}\right| \geq \frac{2^{i-1} \varphi(r)}{2}$, we have

$$
|\hat{f}(x)-\hat{f}(y)|^{n} \int_{2^{i-2} 3 \varphi(r)}^{2^{i} \varphi(r)} \log ^{-l^{2}}\left(\frac{C_{\lambda}\left(t_{0}\right) n I}{\omega_{n-1} t^{n}}\right) \frac{d t}{t} \leq C_{\lambda}(p, n) \int_{A_{i}} J(z, f) d z
$$

for all $x, y \in B_{\varphi(r)}$. For shorter the notation, we write

$$
\alpha=\frac{C_{\lambda}\left(t_{0}\right) n I}{\omega_{n-1}} .
$$

Because

$$
\begin{aligned}
\int_{2^{i-2} 3 \varphi(r)}^{2^{i} \varphi(r)} \frac{d t}{t \log ^{l^{2}}\left(\frac{\alpha}{t^{n}}\right)} & \geq \int_{2^{i-i} \varphi(r)}^{2^{i-2} 3 \varphi(r)} \frac{d s}{\left(s+2^{i-2} \varphi(r)\right) \log ^{l^{2}}\left(\frac{C_{\lambda}\left(t_{0}\right) I n}{\omega_{n-1}\left(s+2^{i-2} \varphi(r)\right)^{n}}\right)} \\
& \geq \frac{1}{3} \int_{2^{i-i} \varphi(r)}^{2^{i-2} 3 \varphi(r)} \frac{d s}{s \log ^{l^{2}}\left(\frac{\alpha}{t^{n}}\right)}
\end{aligned}
$$

we obtain

$$
|\hat{f}(x)-\hat{f}(y)|^{n} \int_{2^{i-1} \varphi(r)}^{2^{i} \varphi(r)} \log ^{-l^{2}}\left(\frac{\alpha}{t^{n}}\right) \frac{d t}{t} \leq C_{\lambda}(p, n) \int_{A_{i}} J(z, f) d z
$$


for all $x, y \in B_{\varphi(r)}$. Summing over the set $S$, we have

$$
|\hat{f}(x)-\hat{f}(y)|^{n} \int_{\varphi(r)}^{r / 2} \log ^{-l^{2}}\left(\frac{\alpha}{t^{n}}\right) \frac{d t}{t} \leq C_{\lambda}(p, n) \int_{B_{r}} J(z, f) d z
$$

for all $x, y \in B_{\varphi(r)}$. Because $I \geq \frac{\omega_{n-1}}{n} r^{n}$, we see that $\frac{n I}{\omega_{n-1} t^{n}} \geq 1$, for all $t \in(0, r)$ and so

$$
\begin{aligned}
\int_{\varphi(r)}^{r / 2} \log ^{-l^{2}}\left(\frac{\alpha}{t^{n}}\right) \frac{d t}{t} & \geq C_{\lambda}^{-l^{2}}\left(t_{0}\right) \int_{\varphi(r)}^{r / 2} \log ^{-l^{2}}\left(\frac{n I}{\omega_{n-1} t^{n}}\right) \frac{d t}{t} \\
& =\frac{C_{\lambda}^{-l^{2}}\left(t_{0}\right)}{\left(l^{2}-1\right) n}\left(1-e^{1-l^{2}}\right) \log ^{-l^{2}+1}\left(\frac{n I}{\omega_{n-1} r^{n}}\right) .
\end{aligned}
$$

Finally recalling the fact $p=n-\frac{1}{2}$ and $t_{0}=t_{0}(p, n, l, \lambda)$ we complete the proof of Lemma 4.3.

Now we complete the proof of the whole theorem (in the case $n>2$ and $l \in\{2, . ., n-1\})$ as follows. Given $x \in B\left(x_{0},(R / 8)^{e}\left[\frac{n I}{\omega_{n-1}}\right]^{\frac{1-e}{n}}\right)$ we consider the ball $B\left(x_{0}, r\right), r=\left[\left|x-x_{0}\right|\left(\frac{n I}{\omega_{n-1}}\right)^{\frac{e-1}{n}}\right]^{\frac{1}{e}}$. By Lemma 4.3, we have

$$
\left|\hat{f}(x)-\hat{f}\left(x_{0}\right)\right|^{n} \leq C_{\lambda, n, l} \log ^{l^{2}-1}\left(\frac{n I}{\omega_{n-1} r^{n}}\right) \int_{B\left(x_{0}, r\right)} J(z, f) d z .
$$

Using Lemma 4.1, we obtain

$$
\int_{B\left(x_{0}, r\right)} J(x, f) d x \leq \frac{C_{\lambda, K, R, n}(\epsilon) \int_{B\left(x_{0}, R\right)} J(z, f) d z}{\log ^{\lambda-\epsilon}\left(\frac{n I}{\omega_{n-1} r^{n}}\right)} .
$$

Combining the inequalities (49) and (50) with the assumptions $\lambda>l^{2}-1$ and $\frac{n I}{\omega_{n-1} r^{n}}=\left(\frac{n I}{\omega_{n-1}\left|x-x_{0}\right|^{n}}\right)^{\frac{1}{e}}$ we finally conclude the desired modulus of continuity (5).

\section{Proof of Theorem 1.2}

In [5, Theorem 1.2], we proved the following result.

Theorem 5.1. Fix $l \in\{1, \ldots, n-1\}, \lambda>l-1$ and $A, B \geq 0$. Let $\mathcal{F}$ be the family of mappings $f: \Omega \rightarrow \mathbb{R}^{n}$ of finite distortion for which

$$
\int_{\Omega} J(x, f) d x \leq A
$$

and

$$
\int_{\Omega} \exp \left(\lambda \sqrt[l]{\mathcal{K}_{l}(x, f)}\right) d x \leq B
$$

Then $\mathcal{F}$ is closed under weak convergence in $W_{\text {loc }}^{1,1}\left(\Omega, \mathbb{R}^{n}\right)$.

Combining this with Ascoli's Theorem and the equicontinuity property of the family $\mathcal{F}$, obtained from Theorem 1.1, the claim follows immediately. 


\section{Proof of Theorem 1.3}

The proof of Theorem 1.3 will be based on the following result.

Theorem 6.1. Let $f \in W_{l o c}^{1, p}\left(\Omega, \mathbb{R}^{n}\right), p>n-1$ be a continuous mapping of finite distortion $K_{O} \in L_{\text {loc }}^{q}(\Omega), q>n-1$. Suppose that (16) holds, and the equation

$$
\int_{\Omega} v(f(x)) J\left(x, f_{1}, . ., f_{i-1}, \varphi, f_{i+1}, \ldots, f_{n}\right) d x=-\int_{\Omega} \varphi(x) \frac{\partial v}{\partial y_{i}}(f(x)) J(x, f) d x
$$

is valid for all $v \in C^{1}\left(\mathbb{R}^{n}, \mathbb{R}^{n}\right)$, each $\varphi \in C_{0}^{\infty}(\Omega)$ and every $i \in\{1, \ldots, n\}$. Then $f$ is either constant or both open and discrete, and $f$ maps sets of measure zero to sets of measure zero.

We refer to [8, Theorem 2.4 and Theorem 3.1] and [9, Theorem 3.2] for the proof of Theorem 6.1. As we are going to appeal to Theorem 6.1, we first observe that $f \in$ $W_{l o c}^{1, p}\left(\Omega, \mathbb{R}^{n}\right)$, for all $[1, n)$ and (16) holds, by Proposition 3.2. Furthermore, using the power series presentation for the function $\exp \left(\lambda K_{O}^{1 / l^{2}}\right)$ and inequality (12), we see that $K_{O} \in L_{l o c}^{q}(\Omega)$, for all $q \in(1, \infty)$. Thus, Theorem 1.3 is immediately from Theorem 6.1 once we verify equation (53).

Fix $\varphi \in C_{0}^{\infty}(\Omega)$ and $i \in\{1, \ldots, n\}$. Pick a domain $\Omega^{\prime}$ so that $\operatorname{spt} \varphi \subset \Omega^{\prime} \subset \subset \Omega$ and denote $\beta=\max \left\{|\nabla v(x)|: x \in \bar{\Omega}^{\prime}\right\}$. We consider the mapping

$$
F_{i}=\left(f_{1}, \ldots, f_{i-1}, \beta f_{i}+v(f), f_{i+1}, \ldots, f_{n}\right) .
$$

By Proposition 3.2 we see that $\left|D^{\sharp} f\right|^{\frac{n}{n-1}} \log ^{-1}\left(e+\left|D^{\sharp} f\right|\right) \in L_{l o c}^{1}(\Omega)$ and so the same is true also to $F_{i}$. Since

$$
J\left(x, F_{i}\right)=\left(\beta+\frac{\partial v}{\partial y_{i}}(f(x))\right) J(x, f) \geq 0
$$

for almost every $x \in \Omega^{\prime}$, we can apply Theorem 1.3 in [1] to conclude that

$$
\int_{\Omega^{\prime}} \varphi(x) J\left(x, F_{i}\right) d x=-\int_{\Omega^{\prime}}\left(\beta f_{i}+v(f)\right) J\left(x, f_{1}, \ldots, f_{i-1}, \varphi, f_{i+1}, \ldots, f_{n}\right) d x .
$$

Furthermore, as (16) holds, we have

$$
\begin{aligned}
\int_{\Omega^{\prime}} \varphi(x) J\left(x, F_{i}\right) d x= & \int_{\Omega^{\prime}} \varphi(x) \frac{\partial v}{\partial y_{i}}(f(x)) J(x, f) d x+\int_{\Omega^{\prime}} \beta \varphi(x) J(x, f) d x \\
= & \int_{\Omega^{\prime}} \varphi(x) \frac{\partial v}{\partial y_{i}}(f(x)) J(x, f) d x \\
& -\beta \int_{\Omega^{\prime}} f_{i} J\left(x, f_{1}, \ldots, f_{i-1}, \varphi, f_{i+1}, \ldots, f_{n}\right)
\end{aligned}
$$

and so (53) follows. All hypothesis of Theorem 6.1 are therefore fulfilled, completing the proof of Theorem 1.3.

Acknowledgement. The author wishes to express his thanks to Professor Pekka Koskela for several useful suggestions and for reading the manuscript. 


\section{References}

1. Giannetti, F., Iwaniec, T., Onninen, J., Verde, A.: Estimates of Jacobians by subdeterminants. J. Geom. Anal. 12, 223-254 (2002)

2. Gehring, F.W.: Rings and quasiconformal mappings in space. Trans. Amer. Math. Soc. 103, 353-393 (1962)

3. Iwaniec, T., Koskela, P., Martin, G. J., Sbordone, C.: Mappings of finite distortion: $L^{n} \log ^{\chi} L$-integrability. J. London Math. Soc. (2) 67, 123-136 (2003)

4. Iwaniec, T., Koskela, P., Onninen, J.: Mappings of finite distortion: Monotonicity and continuity. Invent. Math. 144, 507-531 (2001)

5. Iwaniec, T., Koskela, P., Onninen, J.: Mappings of finite distortion: compactness. Ann. Acad. Sci. Fenn. Math. 27, 391-417 (2002)

6. Iwaniec, T., Martin, G. J.: Geometric Function Theory and Nonlinear Analysis. Oxford Mathematical Monographs. 2001

7. Iwaniec, T., Sbordone, C.: On the integrability of the Jacobian under minimal hypotheses. Arch. Rational Mech. Anal. 119, 129-143 (1992)

8. Kauhanen, J., Koskela, P., Malý J.: Mappings of finite distortion: Discreteness and openness. Arch. Ration. Mech. Anal. 160, 135-151 (2001)

9. Kauhanen, J., Koskela, P., Malý J.: Mappings of finite distortion: Condition N. Condition N. Michigan Math. J. 49, 169-181 (2001)

10. Kauhanen, J., Koskela, P., Malý J., Onninen, J., Zhong, X.: Mappings of finite distortion: Sharp Orlicz-conditions. Rev. Mat. Iberoamericana, to appear.

11. Koskela, P., Malý J.: Mappings of finite distortion: the zero set of the Jacobian. J. Eur. Math. Soc. (JEMS) 5, 95-105 (2003)

12. Koskela, P., Onninen, J.: Mappings of finite distortion: the sharp modulus of continuity. Trans. Amer. Math. Soc. 355, 1905-1920 (2003)

13. Lebesgue, H.: Sur le problème de Dirichlet. Rend. Circ. Palermo 27, 371-402 (1907)

14. Manfredi, J.: Weakly Monotone Functions. The Journal of Geometric Analysis. 3, 393$402(1994)$

15. Morrey, C. B.: On the solutions of quasilinear elliptic partial differential equation, Trans. Amer. Math. Soc., 43, 126-166 (1938)

16. Onninen, J.: A note on the isoperimetric inequality. Proc. Amer. Math. Soc. 131, 3821$3825(2003)$ 